\title{
ESTIMATIVA DA RADIAÇÃO SOLAR GLOBAL À SUPERFÍCIE USANDO UM MODELO ESTOCÁSTICO: CASO SEM NUVENS
}

\author{
Juarez Dantas de Souza', Bernardo Barbosa da Silva² e Juan Carlos Ceballos ${ }^{3}$ \\ Recebido em 5 setembro, 2007 / Aceito em 21 fevereiro, 2008 \\ Received on September 5, 2007 / Accepted on February 21, 2008
}

\begin{abstract}
A radiative transfer model was implemented for a 16-layer stratified atmosphere. The scheme is of a stochastic structure, with a random walk of diffuse photons between transition states until arriving to absorption states within layers, sky or ground. The formalism is associated a first order Markov process. The model exhibits a simple but powerful structure, when compared with other models used for multiple-layered atmospheres. The structure of the model allows the calculation of the planetary reflectance, global radiance in the surface and the absorption in the atmosphere. Transition parameters for each layer are calculated using a delta-SS two-flux approximation. Comparisons with surface measurements and with SBDART code (Santa Barbara DISORT Atmospheric Radiative Transfer), show a good performance of the model when assessing global radiation at ground level for Rayleigh atmospheres as well as for high-loaded aerosol atmospheres during burning season. The precision of the model, in the atmosphere transmittance estimation, makes it important for researchers that act in the area of remote sensing. Main advantages of the model are: simple structure, good accuracy and easy adaptation to an atmosphere composed of an even number of layers.
\end{abstract}

Keywords: Solar radiation, irradiance, radiative transfer, stochastic model.

RESUMO. Neste trabalho implementa-se um modelo de transferência radiativa numa atmosfera estratificada em 16 camadas. 0 esquema tem estrutura estocástica com transições aleatórias de fótons difusos de estados transitórios até um estado de absorção numa camada da atmosfera, no céu ou no solo. 0 formalismo está associado a uma cadeia de Markov de primeira ordem. 0 modelo é uma estrutura simples, mas eficiente quando comparado com outros modelos usados para atmosfera de múltiplas camadas. A estrutura do modelo permite o cálculo da refletância planetária, radiação solar global à superfície e a absorção na atmosfera. Os parâmetros de transição são avaliados mediante aproximação delta-SS de dois fluxos. Verifica-se um bom desempenho do modelo ao se comparar a radiação solar global à superfície com dados medidos à superfície e com o código SBDART (Santa Barbara DISORT Atmospheric Radiative Transfer). A precisão do modelo, na estimativa da transmitância da atmosfera, o torna importante para pesquisadores que atuam na área de sensoriamento remoto. As principais vantagens do modelo utilizado são: estrutura simples, boa precisão e fácil adaptação para uma atmosfera composta por um número par de camadas.

Palavras-chave: irradiância, fluxo radiativo, processo estocástico.

\footnotetext{
${ }^{1}$ Departamento de Matemática e Estatística, Centro de Ciências e Tecnologia - CCT, Universidade Estadual da Paraíba, Rua Juvêncio Arruda, s/n Campus Universitário (Bodocongó), 58109-790 Campina Grande, PB. Tel.-Fax: (83) 3315-3340 - E-mail: juarezd@terra.com.br

2Unidade Acadêmica de Ciências Atmosféricas, Centro de Tecnologia e Recursos Naturais - CTRN, Universidade Federal de Campina Grande - UFCG, Av. Aprígio Veloso, 822, Campus Universitário (Bodocongó), 58109-970 Campina Grande, PB. Tel.-Fax: (83) 3310-1054 - E-mail: bernardo@dca.ufcg.edu.br

${ }^{3}$ Divisão de Satélites e Sistemas Ambientais - DCA, Centro de Previsão de Tempo e Estudos Climáticos - CPTEC, Instituto Nacional de Pesquisas Espaciais - INPE, Rodovia Presidente Dutra km 40, 12630-000 Cachoeira Paulista, SP. Tel.: (12) 3186-9397; Fax: (12) 3101-2835 - E-mail: juanc_ceballos@yahoo.com.br
} 


\section{INTRODUÇÃo}

A radiação solar é uma fonte de energia com muita influência nos processos atmosféricos. As variações no balanço de radiação são fundamentais nos processos atmostéricos e terrestres alterando, por exemplo, a temperatura à superfície, o perfil da taxa vertical de aquecimento e a circulação atmosférica. A propagação da radiação solar é um fenômeno relativamente complexo, em particular quando se pensa na sua possível inclusão em modelos de circulação atmosférica. A propagação monocromática da radiação é descrita pela equação de Schwarzchild (Liou, 1980) e permite descrever detalhadamente a distribuição espacial da absorção e do espalhamento de radiância. Chandrasekhar (1950) desenvolveu uma solução geral exata para a equação de propagação de radiação monocromática numa atmosfera estratificada. Posteriormente, Deirmendjian \& Sekera (1954) aplicaram essa solução a uma atmosfera Rayleigh para determinar a radiação solar global à superfície como função da espessura óptica, do ângulo zenital e do comprimento de onda.

Um método que tem se popularizado nas últimas décadas na solução da equação de transferência radiativa é o método de dois fluxos o qual tem sido objeto de estudo de vários pesquisadores (Liou, 1974; Coakley \& Chylek, 1975; Joseph et al., 1976; Schaller, 1979; Leighton, 1980; Meador \& Weaver, 1980; Zdunkowski et al., 1980; Schmetz, 1984, Filyushkin, et al., 1994). Existem várias versões deste método e todas apresentam praticamente a mesma acurácia (King \& Harshvardhan, 1986). 0 método de dois fluxos tem sido usado para várias finalidades, como exemplo, Fu et al. (1997) para estimar a transferência radiativa no IR e Stephens et al. (2001) para deduzir fluxos de banda larga.

Apesar da grande quantidade de trabalhos, publicados na literatura buscando amenizar a complexidade que envolve a equação de transferência radiativa na atmosfera, ela ainda persiste. Nesse sentido vários pesquisadores (Zorzano et al., 2005; Rozanov et al. 2005; Coelho, 2005; Gueymard, 2008; Subasilar, 2008) têm procurado apresentar novas soluções para a equação de transferência radiativa conforme seja a física do problema a ser resolvido.

Para fins de estimativa da propagação da radiação solar, é suficiente o conhecimento da irradiância (Wm-2). No caso de uma atmosfera estratificada, a equação geral de propagação pode ser reduzida a um par de equações diferenciais ou "método de dois fluxos", cuja solução resulta em um par de irradiâncias, uma ascendente e outra descendente (Ceballos, 1988; Liou, 1992). Os modelos de circulação atmosférica costumam utilizar o esquema de dois fluxos e, genericamente, consideram uma atmosfera com estratificação plano-paralela e cada camada povoada por ar úmido ou por uma nuvem estratiforme (Stephens, 1984; Stephens et al., 2001).

Os esquemas de dois fluxos favorecem a utilização de uma simples descrição estocástica da propagação de radiação solar. Ceballos (1989) aplicou este conceito aos esquemas de dois fluxos para descrever a propagação da radiação na atmosfera. Um modelo estocástico apresenta características simplificadoras do cálculo de fluxos de irradiância e permite a introdução de sistemas descontínuos (como exemplo: campos de nuvens), evidenciando as interações entre os diversos elementos componentes da atmosfera. A determinação de perfis verticais de taxas de aquecimento ou resfriamento, a partir de situações climáticas previamente conhecidas, é uma consequêencia do modelo.

0 presente trabalho objetivou a aplicação do modelo estocástico numa atmosfera estratificada, sem a presença de nuvens, mas incluindo aerossol em região rural e em regiões afetadas por queimadas. A precisão do modelo, na estimativa da transmitância da atmosfera, o torna importante para pesquisadores que atuam na área de sensoriamento remoto. As principais vantagens do modelo utilizado são: estrutura simples, boa precisão e fácil adaptação para uma atmosfera composta por um número par de camadas.

\section{METODOLOGIA}

\section{0 método de dois fluxos}

As aproximações de dois fluxos consistem na solução da equação de transferência radiativa (ETR) por integração do campo de radiância. Para uma atmosfera com profundidade óptica $(\tau)$, essa integração resulta em dois fluxos verticais irradiantes: um proveniente do hemisfério superior [irradiância descendente $E^{\downarrow}(\tau)$ ] e outro do hemisfério inferior [irradiância ascendente $E^{\uparrow}(\tau)$ ], que são estimadas pelo sistema de equações diferenciais (Ceballos, 1988):

$$
\begin{aligned}
\frac{d}{d \tau} E^{\downarrow}(\tau)= & -\alpha_{11} E^{\downarrow}(\tau)+\alpha_{12} E^{\uparrow}(\tau) \\
& +\omega S_{\lambda}(\tau)\left[1-b\left(\mu_{o}\right)\right] \\
\frac{d}{d \tau} E^{\uparrow}(\tau)= & -\alpha_{21} E^{\downarrow}(\tau)+\alpha_{22} E^{\uparrow}(\tau) \\
& -\omega S_{\lambda}(\tau) b\left(\mu_{o}\right)
\end{aligned}
$$

onde $E^{\downarrow}(\tau), E^{\uparrow}(\tau)$ são irradiâncias difusas, $\mu_{0}$ é 0 co-seno do ângulo zenital do Sol $Z_{0}\left(\mu_{0}=\cos Z_{0}\right), \omega$ é 0 albedo simples para espalhamento, os coeficientes $\alpha_{i j}$ são funções do albedo simples, definidos pela Eqs. (7) e (8), $b$ é a fração de retroespaIhamento definida pela Eq. (9) e $S_{\lambda}(\tau)$ o fluxo direcional solar na 
profundidade óptica $\tau$ com evolução estimada pela expressão:

$$
S_{\lambda}(\tau)=S_{0, \lambda} \exp \left(-\tau / \mu_{0}\right) .
$$

Para direção incidente $\Omega$, com interação num elemento de volume e subseqüente espalhamento numa direção $\Omega^{\prime}$ (com ângulo de espalhamento $\Theta$ ), a função de fase para espalhamento pode ser desenvolvida em série de polinômios de Legendre $P_{k}\left(\mu^{*}\right)$, $\mu^{*}=\cos \Theta$ (Liou, 1992). A fração de radiação espalhada que é refletida (retroespalhada) nas direções $\Omega^{\prime}$ do hemisfério oposto a $\Omega$ é avaliada por

$$
b(\Omega)=\frac{1}{4 \pi} \int_{2 \pi} P\left(\Omega_{0}, \Omega^{\prime}\right) d \Omega^{\prime} .
$$

A função de fase tem simetria axial em torno de $\Omega$, de forma que $b(\Omega)$ depende apenas do ângulo zenital de incidência $Z$ e da média azimutal de $P$, de forma que

$$
b(\mu)=\frac{1}{2} \int_{0}^{1} \tilde{P}\left(\mu, \mu^{\prime}\right) d \mu, \mu=\cos Z .
$$

Para funções de fase com baixa assimetria, uma aproximação de primeira ordem para $P(\Theta)$ é apropriada (Shettle \& Weinman, 1970; Liou, 1992):

$$
\tilde{P}\left(\mu_{0}, \mu^{\prime}\right)=1+3 g \mu_{0} \mu^{\prime},
$$

sendo $g$ o fator de assimetria da função de fase, estimado por

$$
g=\frac{1}{4 \pi} \int_{4 \pi} \cos (\Theta) P \cos (\Theta) d \Theta=\frac{C_{1}}{3}
$$

onde $\Theta=$ ângulo de espalhamento com relação à direção de incidência, e $C_{1}$ é 0 coeficiente de primeira ordem no desenvolvimento em série de polinômios de Legendre para a função de fase (Liou, 1992).

Os coeficientes $\alpha_{i j}$ nas Eqs. (1) dependem de propriedades locais de absorção e espalhamento, ponderadas com a distribuição espacial de radiâncias (Ceballos, 1989).

Neste trabalho adota-se o modelo SS ${ }^{1}$, devido à sua simplicidade. Ele supõe que as radiâncias difusas ascendente e descendentes são isotrópicas por hemisfério. Esta aproximação é esperável para baixa anisotropia (por exemplo, para espalhamento Rayleigh) ou no meio de uma nuvem opticamente espessa (após numerosos processos de espalhamento). A hipótese de isotropia hemisférica implica em que as frações de retroespalhamento para radiação difusa ascendente e descendente sejam iguais.
Assumindo um valor médio para a direção de retroespalhamento, $\bar{\mu}$, os coeficientes $\alpha_{i j}$ ficam reduzidos a dois: $\alpha_{11}=\alpha_{22}=\alpha_{1}$ e $\alpha_{12}=\alpha_{21}=\alpha_{2}$ de acordo com as expressões:

$$
\begin{gathered}
\alpha_{1(S S)}=\alpha_{1}=\frac{1-\omega[1-\bar{b}(\mu)]}{\bar{\mu}}, \\
\alpha_{2(S S)}=\alpha_{2}=\frac{\omega \bar{b}(\mu)}{\bar{\mu}} .
\end{gathered}
$$

onde $\bar{b}(\mu)$, estimada pela Eq. (3), tem a forma:

$$
\bar{b}(\mu)=\frac{1}{2}\left(1-\frac{3}{2} g \bar{\mu}\right) .
$$

Para radiância isotrópica $\bar{\mu}=0,5$ e o modelo SS torna-se idêntico ao apresentado por Coakley \& Chylek (1975). Uma solução detalhada do sistema de Eqs. (1) é apresentada no Apêndice A.

\section{Atmosfera multicamada: o modelo estocástico}

A solução apresentada no Apêndice A se refere a uma camada. Seja agora uma atmosfera multicamada com interface nas profundidades ópticas $\tau_{i}, i=0,1,2, \ldots N$, com $\tau_{0}=0$ no topo e $\tau_{N}=\tau_{s}$ à superfície (solo). Ficam assim definidas $N$ camadas horizontais cada uma com espessuras $\Delta \tau_{i}=\tau_{i}-\tau_{i+1}$, para as quais:

a) A espessura óptica total $\tau_{s}$ é obtida conforme a Eq. (10).

$$
\tau_{s}=\sum_{i=0}^{N} \Delta \tau_{i}, \quad i \in\{0,1,2,3, \ldots, N\}
$$

b) Para a i-ésima camada, $M^{\uparrow \downarrow}\left(\tau_{i}\right)$ e $M^{\downarrow \uparrow}\left(\tau_{i+1}\right)$ são irradiâncias difusas emergentes pelo topo e pela base, relativas àquela incidente no seu topo.

c) Estas irradiâncias são soluções das Eqs. (1), de forma que para radiação direta incidente numa camada definese uma transmitância direta $T_{D R}$, difusa $T_{D}$ e total $T_{T}$, refletância $R_{D}$, absortância $A_{D}$, como segue:

$$
\begin{aligned}
& T_{D R}=\exp \left(-m_{0} \Delta \tau\right) ; m_{0}=\mu_{0}^{-1} ; \\
& T_{D}=M^{\downarrow}(\Delta \tau) ; \\
& R_{D}=M^{\uparrow}(\Delta \tau), \\
& T_{T}=T_{D}+T_{D R} ; \\
& A_{D}=1-T_{T}-R_{D} .
\end{aligned}
$$

${ }^{1} 0$ acrônimo SS faz referência a Schuster e Schwarzschild que, no início do século XX utilizaram hipótese simplificadora de isotropia hemisférica na equação geral de propagação (ver Paltridge \& Platt, 1976) 
d) Para radiação incidente difusa, $M^{\uparrow}, M^{\downarrow}$ e $1-M^{\downarrow}-M^{\uparrow}$ avaliam a refletância $R$, transmitância $T$ e absortância $A$ da camada. Note-se que $R, T, A$ diferem dos parâmetros $R_{D}, T_{D}, A_{D}$ (ver Apêndice A).

Um modelo estocástico (ME) proposto por Ceballos (1989) será adotado para resolver 0 sistema de $2 N$ equações que descreve uma atmosfera com $N$ camadas. Nesse ME, a propagação de radiação na atmosfera é concebida como um passeio aleatório de fótons difusos, gerados pela interação da radiação direta com a atmosfera. Essa radiação deposita fótons difusos entre 0 topo e a base da atmosfera, gerando condições iniciais de propagação. 0 passeio aleatório termina com a absorção dos fótons no solo, na atmosfera ou emergindo dela para o espaço. No interior da atmosfera, as propriedades $R, T, A$ são interpretadas como probabilidades de reflexão, transmissão e absorção de fótons difusos, respectivamente. Se um fóton difuso no estado $X_{i}^{\downarrow}$ (descendente no nível $\tau_{i}$ ), emerge da base da camada $i$, os parâmetros $R_{D}, T_{D}$ e $A_{D}$, avaliados na camada $i+1$, descrevem as probabilidades de transição para 0 estado $X_{i+1}^{\downarrow}$ (descendente no nível $\tau_{i+1}$ ), ou $X_{i}^{\downarrow}$ (ascendente no nível $\tau_{i}$ ) ou $A_{i+1}$ (absorvido na camada $i+1$ ). Para um campo de radiâncias difusas onde há isotropia hemisférica, essas probabilidades de transição correspondem às propriedades locais de propagação de radiação difusa e não dependem de que as transiç̧ões

$$
X_{i}^{\downarrow} \rightarrow X_{i+1}^{\downarrow} \quad \text { ou } \quad X_{i}^{\downarrow} \rightarrow X_{i}^{\downarrow}
$$

sejam realizadas pela primeira vez. Em conseqüência, o passeio aleatório é descrito por uma cadeia de Markov de primeira ordem e a solução do sistema de Eqs. (1) permite avaliar probabilidades de transiçã̃o dentro da matriz de Markov correspondente. As probabilidades $R_{D}, T_{D}$ e $A_{D}$ para uma camada não dependem da direção (ascendente ou descendente) da radiação difusa, simplificando assim o cálculo dos resultados. Nesse contexto, a refletância do solo ( $R s)$ é interpretada como a probabilidade de um fóton (proveniente da atmosfera) encontrar-se com a superfície e retornar para a atmosfera, e 1-Rs é a probabilidade de absorção no solo em uma interação.

Na Figura 1 ilustra-se a propagação de radiação difusa para uma atmosfera dividida em 16 camadas homogêneas $(N=16)$. Conforme se observa, as espessuras geométricas das camadas são: $50 \mathrm{~km}$ para a camada 1 (altitude entre 50 e $100 \mathrm{~km}$ ), $10 \mathrm{~km}$ para as camadas 2 e 3 (altitudes entre 30 e $50 \mathrm{~km}$ ), $6 \mathrm{~km}$ para a camada 4 (altitude entre 24 e $30 \mathrm{~km}$ ), e $2 \mathrm{~km}$ para as demais camadas. A partir dos níveis referentes à base e ao topo de cada camada, descreve-se as possíveis posições (estados) que um fóton pode ocupar ao ingressar na atmosfera. Desse modo, caracteriza-se o passeio aleatório que o fóton realiza entre 0 solo e 0 céu. A estrutura apresenta 50 estados possíveis, sendo que os estados de 1 a 18 correspondem a estados absorventes (céu, solo e as 16 camadas) e os demais a estados transitórios. Os estados de número ímpar são descendentes e os de número par ascendentes. Cada camada tem a mesma estrutura de transições ilustrada na Figura 1 para as camadas $1 \mathrm{e} 2$, onde $R, T$, e $A$ correspondem às frações de fluxo já comentadas. 0 estado de número 49 é um estado especial: a posição seguinte da radiação que atinge esse estado é a posição 2 (absorçãa pelo solo, 1 - Rs) ou posição 50 (refletida pelo solo, $R s$ ).

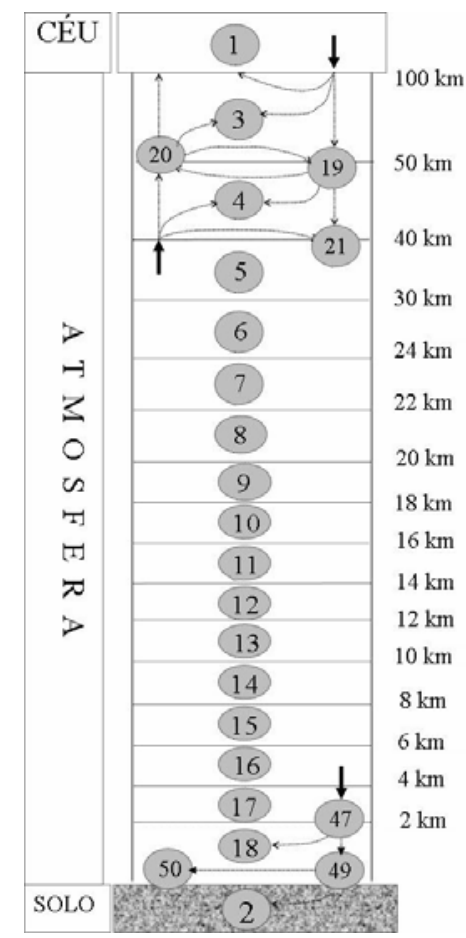

Figura 1 - Estrutura de transições estocásticas para uma atmosfera planoparalela dividida em 16 camadas.

Para o n-ésimo estado corresponde uma probabilidade $p_{n} \mathrm{e}$ 0 conjunto delas define um vetor de estado $P\left\{p_{1}, p_{2}, p_{3}, \ldots\right.$, $\left.p_{n}, \ldots p_{N}\right\} .0$ passeio aleatório dos fótons monocromáticos pelo sistema Terra-atmosfera é descrito por uma seqüência de vetores de estado $P_{0}, P_{1}, P_{2}, \ldots, P_{k}$, relacionados entre si por uma matriz $Q$ de Markov, de forma que:

$$
\begin{gathered}
P_{k}=P_{k-1} Q(n \times n)=P_{0} Q^{k}(n \times n) . \\
\Pi=\lim _{k \rightarrow \infty} P_{k} .
\end{gathered}
$$

Na Eq. (12), o vetor de estado $P_{0}$ tem dimensão $n=3 N+2$, e descreve a probabilidade de produção inicial de fótons difusos 
nos $n$ estados a partir de radiação direta incidente no topo da atmosfera. $Q(n \times n)$ é a matriz de Markov, referente apenas a transiçã̃o ou absorção de radiação difusa, onde suas componentes $q(x, y)$ são as probabilidades da passagem de um estado $x$ para um estado $y$ em apenas uma transição. Na Eq. (13), $P_{k}$ é 0 vetor de estado após $k$ transições e 0 vetor $\Pi$ é interpretado como a posição final (absorção entre 0 solo e 0 céu) que os fótons inicialmente diretos ocupam após seu passeio aleatório pela atmosfera. Fisicamente, o vetor $\Pi$ fornece a distribuição de probabilidades de absorção no sistema Terra-atmosfera, ou seja, $\Pi$ descreve a distribuição vertical de frações absorvidas relativas àquela incidente no topo da atmosfera. Conforme a estrutura do ME, deduz-se que a refletância planetária $(R p)$ e a radiação solar global à superfície $(G)$ correspondem à absorção no céu e no solo, respectivamente.

As Eqs. (12) e (13) evidenciam que 0 caminho percorrido pelos fótons tem duas etapas: a primeira parte da trajetória é percorrida pelo fóton de forma direta até se transformar em difuso (com probabilidade $P_{0}$ ), e a segunda de forma difusa até atingir um estado absorvente. Dessa forma, as componentes de $P_{0}$ podem ser avaliadas como segue.

Para uma atmosfera estratificada como na Figura 1 os estados absorventes são 18, sendo um no céu, um no solo e os demais na atmosfera. Cada camada $i=1,2,3, \ldots, N(N=16)$ com espessura óptica $\Delta \tau_{i}$, tem probabilidade de absorção $\left[A\left(\Delta \tau_{i}\right)\right]$, de transmitância $\left[T\left(\Delta \tau_{i}\right)\right]$ e de refletância $\left[R\left(\Delta \tau_{i}\right)\right]$. Cada camada pode absorver radiação que incide na base ou no topo dela.

Considerando a incidência de radiação direta no topo de uma camada, as probabilidades iniciais referentes aos $n$ estados $P_{0}(n)$ são:

Estados absorventes: 1, 2, 3, ., 18.

$$
\begin{gathered}
P_{0}(1)=R\left(\Delta \tau_{1}\right) . \\
P_{0}(2)=(1-R s) \prod_{i=1}^{16} \exp \left(-m_{0} \Delta \tau_{i}\right) . \\
P_{0}(n)=A\left(\Delta \tau_{i}\right) \prod_{i=1}^{16} \exp \left(-m_{0} \Delta \tau_{i-1}\right), n=i+2 .
\end{gathered}
$$

Estados transitórios: 19, 20, 21, ., 50.

Estados transitórios descendentes:

$$
\begin{gathered}
P_{0}(2 n+1)=\Gamma_{D F}\left(\Delta \tau_{n-8}\right) \prod_{i=9}^{n} \exp \left(-m_{0} \Delta \tau_{i-9}\right), \\
9 \leq n \leq 24 .
\end{gathered}
$$

Estados transitórios ascendentes:

$$
\begin{gathered}
P_{0}(2 n)=R_{D}\left(\Delta \tau_{n-8}\right) \prod_{i=9}^{n} \exp \left(-m_{0} \Delta \tau_{i-9}\right), \\
10 \leq n \leq 24 . \\
P_{0}(50)=R s \prod_{i=1}^{N} \exp \left(-m_{0} \Delta \tau_{i}\right) .
\end{gathered}
$$

Na Eq. (12) a matriz $Q(n \times n)$ está composta por quatro matrizes. As duas primeiras, uma unitária $I(N+2, N+2)$ e outra nula $O(N+2,2 N)$, estão ligadas à absorção em $N+2$ estados; as outras duas são, $U(2 N, N+2)$, descrevendo transições a estados absorventes, e $V(2 N, 2 N)$, que descreve transições entre estados transitórios. 0 limite da potência $Q^{k}$ para $k \rightarrow \infty$ é uma nova matriz $W$ que resulta de uma operação entre as matrizes $U$ e $V$, tal que (Cox \& Miller, 1967):

$$
\begin{gathered}
Q=\left[\begin{array}{cc}
I & O \\
U & V
\end{array}\right], \quad \lim _{k \rightarrow \infty} Q^{k}=\left[\begin{array}{cc}
I & O \\
W & O
\end{array}\right], \\
W=\left(I^{*}-V\right)^{-1} U
\end{gathered}
$$

A estrutura explícita das matrizes $U, V$ e $W$, para 0 caso $N=$ 16, está descrita no Apêndice B.

No modelo estocástico, o resultado da propagação de radiação na atmosfera é determinado pela Eq. (13), de forma que a refletância planetária $\left(R_{P}\right)$, a radiação direta $\left(E_{D R}\right)$, a radiação difusa $\left(E_{D}\right)$, a radiação global à superfície $(G)$ e a irradiância absorvida $\left(E_{A B}\right)$ são estimadas conforme as expressões:

$$
\begin{gathered}
R_{P}=\mu_{0} S_{0} \Pi(1), \\
E_{D R}=\mu_{0} S_{0} \exp \left(-\tau_{s} m_{0}\right)=P_{0}(2), \\
G=\mu_{0} S_{0} \Pi(2) /(1-R s), \\
E_{D}=G-E_{D R}, \\
E_{A B}=1-R p-G .
\end{gathered}
$$

\section{Componentes atmosféricos no modelo}

Neste trabalho, a distribuição vertical dos componentes atmosféricos é feita com base numa atmosfera tropical de McClatchey et al. (1971), que possui um perfil de umidade determinado, concentração de dióxido de carbono de 360 ppm, pressão à superfície igual a 1013 hPa e ozônio com concentração variável com a altura, de aproximadamente 3 ppm perto da superfície, aumentando consideravelmente na estratosfera (máximo de 18 ppm aos 
$25 \mathrm{~km}$ ) e caindo para 2,14 $\times 10^{-6} \mathrm{ppm}$ acima de $100 \mathrm{~km}$ e coluna total em torno de $300 \mathrm{DU}$.

Os conjuntos de parâmetros espectralmente discretos referentes a aerossol, ozônio e vapor d'água, assim como os valores da irradiância solar no topo da atmosfera, foram representados por funções contínuas obtidas através de ajustes de curvas.

\section{Atmosfera limpa e seca}

Adota-se como "atmosfera limpa e seca" uma atmosfera sem aerossóis e vapor d'água. Essa atmosfera é uma mistura de oxigênio $\left(\mathrm{O}_{2}\right)$, nitrogênio $\left(\mathrm{N}_{2}\right)$ e dióxido de carbono $\left(\mathrm{CO}_{2}\right)$ como gases permanentes, com concentração relativa constante com a altura. 0 ozônio é também um gás permanente, mas com concentração variável com a altura. Retirando $0 \mathrm{O}_{3}$ no visível e infravermelho próximo, essa atmosfera limpa e seca deixa de ser absorvente (albedo simples $\omega=1$ ), de forma que a radiação incidente é apenas espalhada e segue um padrão cuja teoria foi descrita por J.W. Strutt (Lord Rayleigh) em 1871 (McCartney, 1976). Essa atmosfera é conhecida como "atmosfera Rayleigh". No espalhamento Rayleigh para radiação não polarizada (como a solar incidente), a função de fase para espalhamento com ângulo $\Theta$ tem a forma:

$$
P(\mu *)=\frac{3}{4}\left(1+\mu *^{2}\right), \mu *=\cos (\Theta) .
$$

Dada a simetria $g=0$ da função de fase, a fração de retroespaIhamento vale 0,5 para todo ângulo de incidência da radiação, ou seja, $b\left(\mu_{o}\right)=b(\mu)=0,5$. Assim, os coeficientes $\alpha_{i}$ referentes aos modelos de dois fluxos para atmosfera Rayleigh têm 0 mesmo valor $\left(\alpha_{i}=\alpha\right)$.

Numa atmosfera limpa e seca, a espessura óptica, $\Delta \tau_{a r}$, de uma camada com espessura (em pressão) $\Delta P$ é calculada conforme a expressão (Paltridge \& Platt, 1976):

$$
\Delta \tau_{a r}=\frac{\Delta P}{P_{\text {sup }}} \cdot 0,0088 \lambda^{-4,08}
$$

onde $\lambda$ é 0 comprimento de onda em $\mu \mathrm{m}$ e $P_{\text {sup }}$ a pressão atmosférica à superfície em $\mathrm{hPa}$.

\section{Ozônio $\left(\mathrm{O}_{3}\right)$}

No espectro solar, 0 ozônio apresenta as bandas de absorção de Hartley $(\lambda<0,31 \mu \mathrm{m})$, de Huggins $(0,313<\lambda<0,340 \mu \mathrm{m})$ e de Chappuis $(0,45<\lambda<0,76 \mu \mathrm{m})$. Considera-se que a banda de Hartley (muito intensa) seja totalmente absorvente no seu intervalo espectral.
A profundidade óptica referente ao ozônio $\left(\tau_{3}\right)$ pode ser calculada usando a expressão:

$$
\tau_{3}=\beta_{O_{3}} \cdot \delta_{O_{3}}
$$

onde $\beta_{O_{3}}$ é 0 coeficiente de absorção e $\delta_{O_{3}}$ a quantidade (na coluna atmosférica) do gás em cm-CNTP. É importante notar que as bandas de absorção do ozônio são espectralmente contínuas, de forma que a Lei de Beer se verifica para qualquer comprimento de onda. Com os valores de $\beta_{O_{3}}$ tabelados por Leckner (1978), foi feito um ajuste de curvas, apresentadas na Tabela 1.

A profundidade óptica $(\tau)$ e 0 albedo simples $(\omega)$, resultantes da mistura entre ar e ozônio numa camada com espessura $\Delta P$, são avaliados conforme as expressões:

$$
\begin{gathered}
\tau=\Delta \tau_{a r}+\beta_{O_{3}} \delta_{O_{3}}, \\
\omega=\frac{\Delta \tau_{a r}}{\Delta \tau_{a r}+\beta_{O_{3}} \delta_{O_{3}}}
\end{gathered}
$$

Neste trabalho, 0 ozônio é distribuído nas camadas acima de 8 km de acordo a concentração em uma atmosfera tropical.

\section{Aerossol}

Os aerossóis exercem forte influência nos fluxos radiativos de ondas curtas. A falta de informações sobre os parâmetros de aerossóis, profundidade óptica, fator de assimetria e albedo simples, contribui para aumentar a incerteza nas mudanças do clima global. Para alguns locais, a exemplo de Cuiabá - MT (15,739 S; 56,021 W; 210 m) no Centro Oeste do Brasil, existem informações sobre parâmetros de aerossóis, e que podem ser encontradas no site da AERONET (AErosol RObotic NETwork: <http://aeronet.gsfc.nasa.gov/>, acessado em 10/04/2007). Já para outros locais existem informações apenas sobre a profundidade óptica, disponíveis através de imagens do sensor MODIS (MODerate resolution Imaging Spectroradiometer) a bordo do satélite Terra e Aqua, produto MOD04 (específico para aerossol) ou MOD08 no site: < http://ladsweb.nascom.nasa.gov/ data/search.html>, acessado em 15/06/2007. É grande a diversidade de tipos de aerossóis e a sua variabilidade temporal. Isso dificulta uma parametrização geral para transferência radiativa. No que segue descreve-se o procedimento para estimar os parâmetros de aerossóis referentes à profundidade óptica $\left(\tau_{a}\right)$, albedo simples $\left(\omega_{a}\right)$ e fator de assimetria $\left(g_{a}\right)$, usados neste trabalho. 
Tabela 1 - Coeficiente de absorção para ozônio no ultravioleta (banda de Huggins) e no visível (banda de Chappuis).

\begin{tabular}{|c|l|}
\hline $\begin{array}{c}\text { Intervalo espectral } \\
\left(\lambda_{1} ; \lambda_{2}\right)(\mu \mathrm{m})\end{array}$ & Coeficiente de absorção $\beta_{O_{3}}(\lambda)[\mathrm{cm}-\mathrm{CNTP}]^{-1}$ \\
\hline$(0,300 ; 0,315]$ & $\beta_{O_{3}}(\lambda)=\exp \left(174,4-996,67 \lambda+1410,74 \lambda^{2}\right)$ \\
\hline$(0,315 ; 0,35]$ & $\beta_{O_{3}}(\lambda)=\exp \left(-5+164,17 \lambda-468,35 \lambda^{2}\right)$ \\
\hline$(0,450 ; 0,565]$ & $\beta_{O_{3}}(\lambda)=2,5-2,243 \lambda^{-1}+0,504 \lambda^{-2}$ \\
\hline$(0,565 ; 0,605]$ & $\begin{array}{r}\beta_{O_{3}}(\lambda)=-246109,53+714306,2652 \lambda^{-1}-828956,4 \lambda^{-2} \\
+480816,2554 \lambda^{-3}-139388,0532 \lambda^{-4}+16156,957 \lambda^{-5}\end{array}$ \\
\hline$(0,605 ; 0,79]$ & $\beta_{O_{3}}(\lambda)=\exp \left(-18,253+65,0446 \lambda-63,283 \lambda^{2}\right)$ \\
\hline
\end{tabular}

A profundidade óptica $\left(\tau_{a}\right)$ é avaliada pela integral:

$$
\begin{aligned}
\tau_{a}(\lambda) & =\int_{0}^{\infty}\left[\int_{0}^{r} \sigma(r, \lambda) n(r, z) d r\right] d z \\
& =\int_{0}^{\infty} \beta(\lambda, z) d z \\
& =\beta(\lambda, 0) \int_{0}^{\infty} \frac{n(z)}{n(0)} d z
\end{aligned}
$$

ou seja,

$$
\tau_{a}(\lambda)=\beta(\lambda, 0) \int_{0}^{\infty} \frac{n(z)}{n_{o}(z)} d z .
$$

Na Eq. (30), $\sigma$ é a seção efetiva de uma partícula de aerossol com raio $r$ para radiação com comprimento de onda $(\lambda)$, e $n(r, z)$ a densidade espectral de tamanhos de partículas na altitude $z$. Para uma dada propriedade físico-química do aerossol, pode-se avaliar os coeficientes de atenuação e absorção e outros parâmetros para condições de superfície. A Eq. (31) sugere que a espessura óptica relativa a um certo $\lambda$ pode ser avaliada definindo-se 0 valor absoluto mediante a estimativa de $\tau$ para apenas um comprimento de onda (usualmente $\lambda=0,55 \mu \mathrm{m}$ ). Neste trabalho a espessura óptica é estimada conforme a expressão:

$$
\tau(\lambda)=\tau(\lambda=0.55)\left(\frac{0.55}{\lambda}\right)^{\alpha}
$$

onde $\alpha$ é 0 expoente de Angström.

Partículas cujas dimensões são comparáveis com 0 comprimento de onda da radiação (aerossol, gotas de água em nuvens) têm fator de assimetria elevado. Nessas condições, a aproximação de primeira ordem da função de fase, estimada pela Eq. (4), não é mais adequada. Para compensar os efeitos de uma elevada assimetria, a função de fase é submetida a uma "aproximação- $\delta$ ", gerando o modelo $\delta$-SS. A introdução da "aproximação- $\delta$ " resulta em uma nova função de fase, correspondente a soma de uma função $\delta$ de Dirac com peso (ponderação) $f$ e uma função de fase remanescente $P^{\prime}\left(\mu, \mu^{\prime}\right)$ com menor assimetria e peso $1-f$, de forma que:

$$
\begin{aligned}
P\left(\mu, \mu^{\prime}\right) & =2 f \delta\left(\mu, \mu^{\prime}\right)+(1-f) P^{\prime}\left(\mu, \mu^{\prime}\right) \\
& =2 f \delta\left(\mu, \mu^{\prime}\right)+(1-f)\left(1+g^{\prime} \mu \mu^{\prime}\right) .
\end{aligned}
$$

Combinando as Eqs. (6) e (33) para uma camada composta apenas de aerossol com fator de assimetria $g_{a}$, obtém-se o fator de assimetria efetivo $g_{a}^{\prime}$ ajustado à função $\delta$ de Dirac conforme a expressão:

$$
g_{a}^{\prime}=\frac{g_{a}-f}{1-f}
$$

A introdução da "aproximação- $\delta$ " implica em conservar formalmente 0 sistema de Eqs. (1), mas efetua-se uma mudança de escala nos parâmetros ópticos $\left(\tau_{a}, g_{a}, \omega_{a}\right) \rightarrow\left(\tau_{a}^{\prime}, g_{a}^{\prime}, \omega_{a}^{\prime}\right)$ (Liou, 1992). Para um espalhamento devido apenas ao aerossol os parâmetros $\tau_{a}^{\prime}$ e $\omega_{a}^{\prime}$ são introduzidos no modelo conforme as expressões:

$$
\begin{gathered}
\tau_{a}^{\prime}=\left(1-\omega_{a} f\right) \tau_{a} ; \\
\omega_{a}^{\prime}=\frac{\omega_{a}(1-f)}{1-\omega_{a} f}
\end{gathered}
$$

Uma aproximação para o peso $f$ é feita aproximando a função de fase em série de polinômios de Legendre através da função de fase de Henyey-Greenstein (Joseph et al., 1976; Liou, 1992). Dessa aproximação resulta um valor de $f$ tal que

$$
f=g_{a}^{2}
$$

A carga de aerossol é distribuída ate a altura de $4 \mathrm{~km}$, resultando numa mistura de "ar" ( $\left.\omega_{a r}=1, \tau_{a r}, g_{a r}=0\right)$ e aerossol de forma que a espessura óptica, 0 albedo simples e o fator de 
assimetria da camada ( $\tau, \omega, g$ ) são avaliados por:

$$
\begin{gathered}
\tau=\tau_{a}^{\prime}+\tau_{a r} \\
\omega=\frac{\left(\omega_{a r} \tau_{a r}+\omega_{a}^{\prime} \tau_{a}^{\prime}\right)}{\tau} \\
g=\frac{\left(g_{a r} \tau_{a r}+g_{a}^{\prime} \tau_{a}^{\prime}\right)}{\tau}=\frac{g_{a}^{\prime} \tau_{a}^{\prime}}{\tau}
\end{gathered}
$$

Conforme a Eq. (3), a fração de retroespalhamento decorrente da mistura para a radiação direta $b\left(\mu_{0}\right)$ é:

$$
b\left(\mu_{0}\right)=\frac{1}{2}\left(1-\frac{3}{2} g \mu_{0}\right)
$$

e a fração média de retroespalhamento para radiação difusa é dada por:

$$
\bar{b}=\frac{1}{2}\left(1-\frac{3}{4} g\right)
$$

\section{Vapor d'água}

A parametrização da absorção por vapor d'água é fundamental nos processos de transferência radiativa (Chou \& Suarez, 1999; Zhang et al., 2005). As bandas de absorção do vapor d'água com centro em $0,72,0,8,0,94,1,1,1,38,1,87$ e $2,7 \mu \mathrm{m}$ não permitem um tratamento monocromático que possibilite a aplicação da Lei de Beer. Alguns modelos empíricos, com base em dados experimentais, estabelecem valores numéricos de coeficientes de absorção. Leckner (1978), a partir de dados de McClatchey et al. (1971), propôs a seguinte expressão para transmitância da radiação solar no nível da superfície,

$$
\begin{gathered}
T_{H_{2}} O\left[\beta_{w}(\lambda), w\right] \\
=\exp \left[-\frac{0,2385 \mu_{0} \beta_{w}(\lambda) w}{\left(1+20,07 \mu_{0} \beta_{w}(\lambda) w\right)^{0.45}}\right]
\end{gathered}
$$

onde $\beta_{w}(\lambda)$ são coeficientes espectrais tabelados (Iqbal, 1983; Lenoble, 1993) e $w$ é 0 total de água precipitável na atmosfera. Neste trabalho os valores de $\beta_{w}(\lambda)$ são estimados conforme expressões constantes na Tabela 2 que foram obtidas a partir dos valores tabelados, e a transmitância é definida na forma;

$$
\begin{gathered}
T_{H_{2} O}\left[\beta_{w}(\lambda), w\right] \\
=\exp \left[-\frac{0,24 \beta_{w}(\lambda) w}{\left(1+20,07 \beta_{w}(\lambda) w\right)^{0.45} \mu_{0}}\right]
\end{gathered}
$$

\section{Radiação solar global à superfície}

A radiação solar global à superfície $(G)$ na faixa espectral de 0,3 a 3,0 $\mu \mathrm{m}$ é estimada conforme a expressão:

$$
G=D_{T S} \int_{0,3}^{3} \mu_{0} S_{0}(\lambda) G(\lambda) T_{H_{2} O}(\lambda, w) d \lambda
$$

Nesta equação, $D_{T S}$ é 0 fator de correção de $S_{o}$ por excentricidade da órbita terrestre, $G(\lambda)$ se estima pelo modelo estocástico (Eq. 22) e $T_{\mathrm{H}_{2} \mathrm{O}}$ é avaliada pela Eq. (44). 0 produto $G \cdot T_{\mathrm{H}_{2} \mathrm{O}}$ é permissível, uma vez que no espectro visível não há absorção pelo vapor $\left(T_{\mathrm{H}_{2} \mathrm{O}}=1\right)$ e no infravermelho solar o espalhamento tem uma importância de ordem muito inferior a da absorção, especialmente numa atmosfera com carga de aerossol muito baixa. A eficiência destas parametrizações é testada na seção seguinte.

\section{RESULTADOS E DISCUSSÃO}

Nesta seção é feita uma aplicação das parametrizações apresentadas nas seções anteriores. A irradiância solar espectral no topo da atmosfera tem como referência o espectro de Frölich Wehrli (Iqbal, 1983). Inseriu-se no modelo um conjunto de funções contínuas que permite integrar os parâmetros atmosféricos com resolução $d \lambda=0,005 \mu \mathrm{m}$. Nas comparações feitas entre o ME e o SBDART (Ricchiazzi et al., 1998) são usados os mesmos parâmetros de entrada (pressão atmosférica à superfície, localização, hora, $0_{3}$, $\mathrm{H}_{2} \mathrm{O}$ e aerossol). No SBDART, usa-se uma aproximação $\delta$-M e a função de fase de Henyey-Greenstein (SBDART, 2004).

0 desempenho do modelo é observado em situações bastante específicas: a) com a fórmula de Lacis \& Hansen (1974), b) numa zona rural de Quixeré - CE (5,07 S; 37,86 W; 130 m), onde a atmosfera é bastante limpa apresentando baixa carga de aerossol e c) numa região de queimadas, Cuiabá - MT (15,739 S; 56,021 W; $210 \mathrm{~m}$ ), onde a carga de aerossol é bastante elevada.

\section{Comparação com a fórmula de Lacis \& Hansen (1974)}

Com um erro de aproximadamente $1 \%$, a fórmula de Lacis \& Hansen (1974), Eq. (46), estima a $R p$ para todo espectro solar numa atmosfera plano-paralela com dispersão Rayleigh, perfil padrão de ozônio e superfície com refletância nula.

$$
R p=\frac{0,28}{\left(1+0,643 \mu_{0}\right)} .
$$

Neste trabalho, para comparar resultados entre a fórmula de Lacis \& Hansen (1974) e 0 ME foi adotada uma carga de $\mathrm{O}_{3}=$ $300 \mathrm{DU}$ e $\mathrm{P}_{0}=950 \mathrm{mb}$. A $R p$ no ME é obtida por integração espectral com resolução de $0,005 \mu \mathrm{m}$ no espectro de 0,3 a 
Tabela 2 - Coeficientes espectrais $\beta_{w}(\lambda)$ relativos à absorção por vapor d'água.

\begin{tabular}{|c|l|}
\hline $\begin{array}{c}\text { Intervalo espectral } \\
\left(\lambda_{1} ; \lambda_{2}\right)(\mu \mathrm{m})\end{array}$ & Coeficiente $\beta_{w}(\lambda)$ \\
\hline$(0,69 ; 0,72]$ & $\beta_{w}(\lambda)=-0,004 /\left(1-2,84 \lambda+2 \lambda^{2}\right)$ \\
\hline$(0,72 ; 0,76]$ & $\beta_{w}(\lambda)=\exp \left(-6606,7+18243 \lambda-12590 \lambda^{2}\right)$ \\
\hline$(0,76 ; 0,86]$ & $\beta_{w}(\lambda)=\exp \left(-7785+18955,667 \lambda-11538 \lambda^{2}\right)$ \\
\hline$(0,86 ; 1,00]$ & $\beta_{w}(\lambda)=\exp \left(-2676+5671 \lambda-3000 \lambda^{2}\right)$ \\
\hline$(1,00 ; 1,20]$ & $\beta_{w}(\lambda)=\exp \left(-2378+4198 \lambda-1848,6 \lambda^{2}\right)$ \\
\hline$(1,20 ; 1,60]$ & $\beta_{w}(\lambda)=\exp \left(-1077,5+1551 \lambda-554 \lambda^{2}\right)$ \\
\hline$(1,60 ; 1,85]$ & $\beta_{w}(\lambda)=\exp \left(-527,97+529 \lambda-129 \lambda^{2}\right)$ \\
\hline$(1,85 ; 2,50]$ & $\beta_{w}(\lambda)=\exp \left(384-349 \lambda+79,5 \lambda^{2}\right)$ \\
\hline$(2,50 ; 2,80]$ & $\beta_{w}(\lambda)=\exp \left(-476+363 \lambda-68 \lambda^{2}\right)$ \\
\hline$(2,80 ; 3,30]$ & $\beta_{w}(\lambda)=\exp \left(410,434-258,361 \lambda+41,067 \lambda^{2}\right)$ \\
\hline
\end{tabular}

$3,0 \mu \mathrm{m}$ para diferentes valores de $\mu_{o}(0,2,0,4,0,6,0,8,1)$. 0 resultado mostrado na Figura 2 apresenta um erro em relação ao ME que varia entre $1,9 \%$ para $\mu_{0}=0,2$ e $2,5 \%$ para $\mu_{0}=1$. Essa pequena margem de erro mostra que nestas condições, 0 ME é eficiente no cômputo da $R p$ e que através do mesmo a $R p$ pode ser estimada espectralmente.

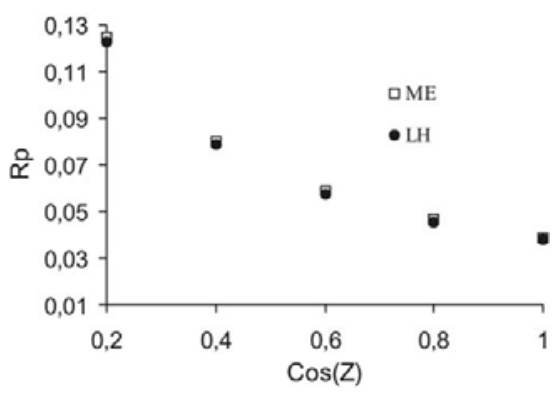

Figura 2 - Comparação entre o ME e a fórmula de Lacis \& Hansen (1974) no cômputo da refletância planetária $(R p)$ em função do ângulo de incidência.

\section{Resultados para uma região rural [Quixeré - CE (5,07 S; 37,86 W; 130 m)]}

Fez-se uma comparação com medições realizadas em 16/09/ 2005, na área rural (irrigada) na cidade de Quixeré. Este dia foi escolhido por apresentar condições de céu claro. As medidas de superfície foram obtidas através de um saldo-radiômetro (CNR1 da Campbell Inc.) e de um analisador de $\mathrm{CO}_{2}$ e $\mathrm{H}_{2} \mathrm{O}$ (LI-7500 da LI-COR). 0 CNR1 fornece dados de radiação solar, incidente (medida por um piranômetro, CM3, direcionado para cima) e refletido pela superfície (medida por um piranômetro, CM3, direcionado para baixo), no espectro de 0,305 a $2,8 \mu \mathrm{m}$ com uma precisão de 2,5\%, e o LI-7500 fornece a temperatura do ar (Ta), umidade relativa (UR), pressão atmosférica $\left(\mathrm{P}_{0}\right)$ e concentração de $\mathrm{H}_{2} \mathrm{O}$.

Nesta aplicação foi usada uma carga de ozônio de 270 DU. Os parâmetros de aerossol $\tau(0.55)=0,12$ e $\alpha=0,63$ na Eq. (32) e água precipitável na atmosfera $w=2,4 \mathrm{~g} . \mathrm{cm}^{-2}$ foram obtidos através do produto MOD08, e por falta de informações os parâmetros $\omega$ e $g$ foram adotados como $\omega=0.93$ e $g=0.64$ com base no perfil de aerossol rural de Shettle \& Fenn (1979), usado no SBDART.

Para avaliar as parametrizações referentes ao $\mathrm{O}_{3}$ e ao $\mathrm{H}_{2} \mathrm{O}$ fez-se um teste na estimativa da transmitância total da atmosfera usando como parâmetros de entrada: $\mu_{0}=0,623, P_{0}=970 \mathrm{mb}$, $w=2,4$ g. $\mathrm{cm}^{2}{ }^{2}$, carga de ozônio $=270 \mathrm{DU}$ e aerossol $[\tau(0.55)$ $=0,12, \alpha=0,63, \omega=0.93, g=0.64]$.

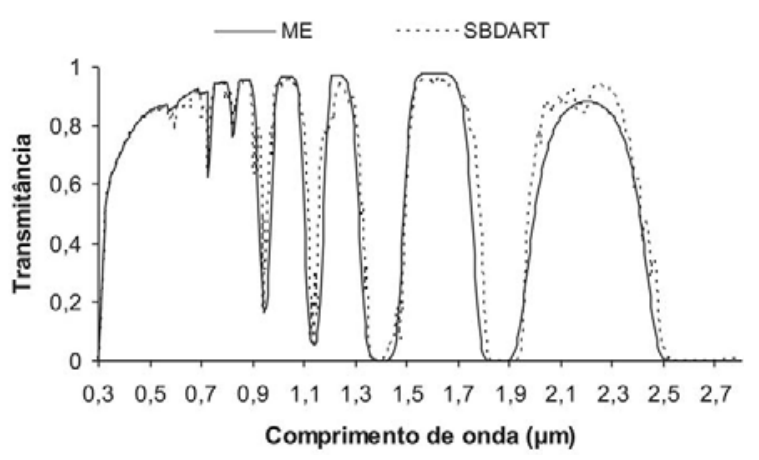

Figura 3 - Transmitância numa atmosfera rural onde: $\mu_{0}=0,623, w=$ $2,4 \mathrm{~g} . \mathrm{cm}^{-2}, \mathrm{P}_{0}=970 \mathrm{mb} ; \mathrm{O}_{3}=270 \mathrm{DU}, \tau_{a}(0,55)=0,12$, expoente de Angström $\alpha=0,63 ; \omega=0,93$ e $g=0,64$. Comparação entre o ME e 0 SBDART.

Na Figura 3 mostra-se o resultado para a transmitância espectral total da atmosfera, onde co-seno do ângulo zenital do sol 
$\mu_{0}=0,623$ e $\mathrm{P}_{0}=970 \mathrm{mb}$. A maior diferença entre $0 \mathrm{ME}$ e 0 SBDART ocorre na faixa espectral entre 2 e $2,3 \mu \mathrm{m}$ onde o fluxo incidente no topo da atmosfera é menor que 1,9\% da constante solar, de forma que o erro decorrente dessa diferença é de pouca importância, e pode ser constatado na Figura 4, que ilustra o ciclo diário da radiação global.

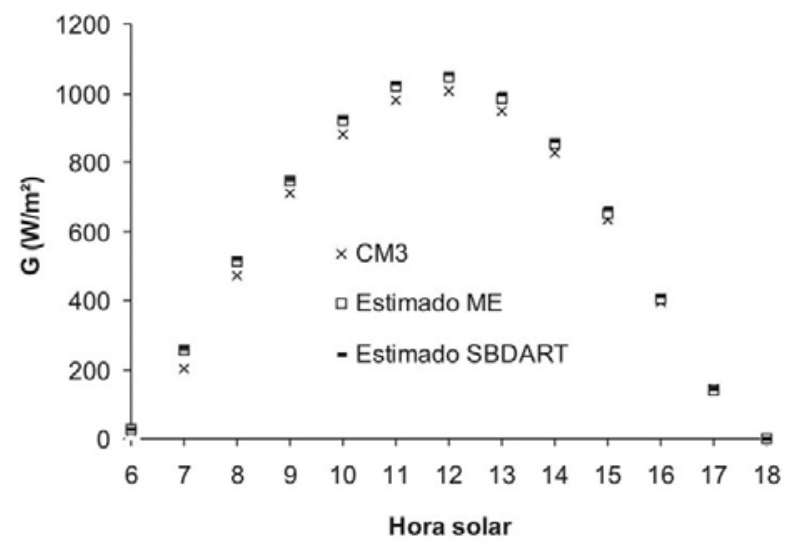

Figura 4 - Ciclo diário da $\mathrm{G}\left(\mathrm{W} / \mathrm{m}^{2}\right)$ para 0 dia 16/09/2005 em Quixeré onde: $w=2,4 \mathrm{~g} \cdot \mathrm{cm}^{-2}, \mathrm{P}_{0}=970 \mathrm{mb}, \tau_{a}(0.55)=0,12$, expoente de Angström 0,63, $\omega=0,93$ e $g=0,64$, albedo da superfície ao meio dia solar $=0,15$. Valor exato medido pelo piranômetro CM3 $(\times)$, estimado pelo ME $(\square)$, estimado pelo SBDART (-).

Na Figura 4 mostra-se 0 ciclo diário da radiação solar global $\left(\mathrm{Wm}^{-2}\right)$ à superfície medida pelo piranômetro CM3 $(x)$ (amostrada a cada 10 minutos), comparada com as estimadas pelo ME $(\square)$ e SBDART (-). Os parâmetros de aerossol e água precipitável são os mesmos descritos no primeiro parágrafo desta seção. 0 albedo do solo ao meio dia era de 0,15. Entre 9 e 16 horas solar o comportamento do ME difere do SBDART em menos de 1,3\% para irradiância medidas entre 600 e $1000 \mathrm{Wm}^{-2}$. As diferenças dos dois modelos com relação às medições são de 3,86\% (ME) e 5,02\% (SBDART). Ressalta-se ainda, que 0 ME e o SBDART estimam valores instantâneos enquanto os valores obtidos pelo CNR1 correspondem à média a cada $10 \mathrm{mi}$ nutos. Assumiu-se que os parâmetros de aerossol $\omega, \tau_{a}$ e $g$ eram constantes ao longo do dia e em todo espectro solar, eles podem ter sido adequados para a parte da tarde e não para a parte da manhã conforme desempenho do ME e do SBDART mostrado na Figura 4. Também é possível que a carga de aerossol, usada nesta aplicação, fornecida pela imagem MOD08, que tem uma resolução de um grau, não tenha sido adequada para o local onde se realizou as medidas de superfície.

\section{Resultados para uma região de queimadas} [Cuiabá-Miranda - MT (15,739 S; 56,021 W; 210 m)]

Nesta aplicação utilizou-se dados referentes à estação de CuiabáMiranda. Foi escolhido o dia 06/09/2005 por apresentar características de céu claro. Os parâmetros de aerossol, albedo simples, fator de assimetria, espessura óptica e vapor d'água foram consultadas no site da AERONET. Os dados de radiação solar foram obtidos na rede SolRad-Net (Solar Radiation Network) disponível no site: < http://solrad-net.gsfc.nasa.gov> , acessado em 10/12/2006 e os dados de pressão e temperatura foram consultados em <http://tempo.cptec.inpe.br:9080/PCD/>, acessado em 10/12/2006. Para o dia em análise observaram-se os seguintes dados: $\tau(0,55)=1,93$, expoente de Angström $\alpha=1,87$, valor médio do albedo simples e do fator de assimetria respectivamente iguais a 0,94 e 0,58, água precipitável $w=3,26 \mathrm{~g} . \mathrm{cm}^{2}$, temperatura do ar igual a $300 \mathrm{~K}$, pressão $980 \mathrm{mb}$ e umidade relativa de $60 \%$. Com base no produto MOD09, o albedo da superfície no momento da passagem do satélite (11 horas solar) era de 0,14. A variação do albedo da superfície com 0 ângulo zenital foi feita conforme Paltridge \& Platt (1976).

Na Figura 5 mostra-se a transmitância da atmosfera no instante em que $\mu_{0}=0,797$ (10 horas solar). Assim como no caso da seção 3.2 observa-se um bom comportamento das parametrizações referentes ao $\mathrm{O}_{3}$ e ao $\mathrm{H}_{2} \mathrm{O}$.

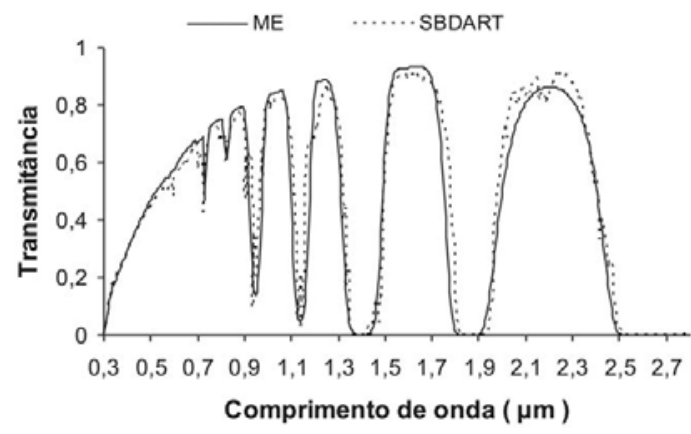

Figura 5 - Transmitância numa região de queimadas onde: $\mu_{0}=0,797, w=$ $3,26 \mathrm{~g} . \mathrm{cm}^{-2}, \mathrm{P}_{0}=980 \mathrm{mb}, 0_{3}=270 \mathrm{DU}, \tau_{a}(0,55)=1,93$, expoente de Angström $\alpha=1,87, \omega=0,94, g=0,58$. Comparação entre o ME e 0 SBDART.

Na Figura 6, mostra-se o ciclo diário da radiação solar global $\left(\mathrm{Wm}^{-2}\right)$ à superfície medida pelo piranômetro da rede SolRadNet (amostrada a cada 2 minutos), Valor exato $(\times)$, comparada com as estimadas pelo ME ( $\square$ ) e SBDART ( - ). No intervalo de 8 às 12 horas solar o ME comete um erro médio de 3,6\% e o SBDART $1,7 \%$ e no intervalo de 12 às 16,5 horas solar os erros são, respectivamente, $-1,9 \%$ e $-4,4 \%$. Nesse caso, no intervalo de 8 às 16,5 horas solar, 0 ME mostra-se mais eficiente que 0 SBDART 
apresentando um erro médio de 0,86\% enquanto o SBDART apresenta um erro de $-1,4 \%$.

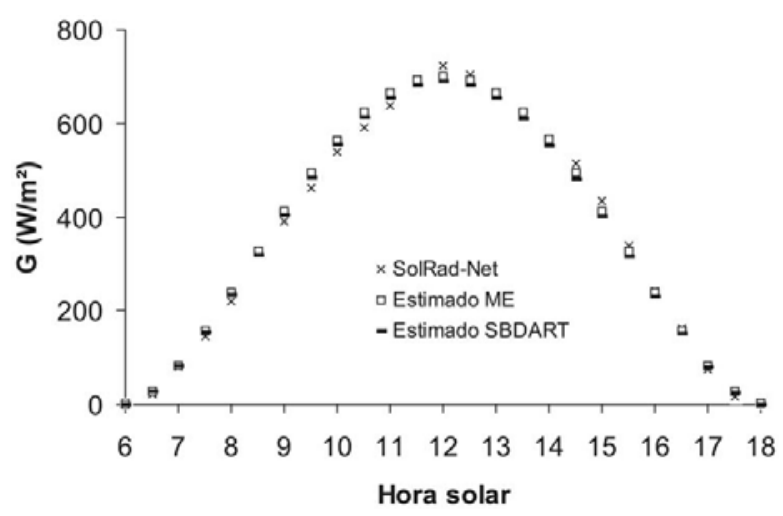

Figura 6 - Ciclo diário da $\mathrm{G}\left(\mathrm{W} / \mathrm{m}^{2}\right)$ para 0 dia 06/09/2005 em Cuiabá-Miranda onde: $w=3,26 \mathrm{~g} . \mathrm{cm}^{-2}, \mathrm{P}_{0}=980, \tau_{a}(0,55)=1,93$ e expoente de Angström $\alpha=1,87, \omega=0,94, g=0,58$, albedo da superfície às 11 horas solar $=0,14$. Valor exato $(\times)$, estimado pelo ME $(\square)$, estimado pelo SBDART ( - ).

\section{CONCLUSÕES}

0 modelo proposto apresenta uma estrutura simples e permite estimar a irradiância que chega a superfície com boa precisão ao mesmo tempo em que avalia o perfil vertical de absorção na atmosfera. Os principais parâmetros envolvidos no modelo são: ozônio, vapor d'água, aerossol, ângulo zenital do Sol e pressão a superfície. Os resultados das aplicações ilustrados nas Figuras 4 e 6 mostram que o ME tem um bom desempenho na estimativa da radiação solar global à superfície em condições de céu claro chegando a ser ligeiramente melhor que o SBDART, tanto na região com baixa carga de aerossol (Quixeré - Fig. 4) como na região com elevada carga de aerossol (Cuiabá-Miranda Fig. 6). Pode-se concluir que a estrutura estocástica proposta por Ceballos (1989) é adequada para estratificar uma atmosfera em um número par de camadas, e que as parametrizações implantadas para absorção pelo vapor d'água e pelo ozônio constituem uma boa alternativa para integração de fluxos monocromáticos.

\section{AGRADECIMENTOS}

Os autores agradecem à Universidade Estadual da Paraíba pela liberação do primeiro autor para cursar o doutorado em Meteorologia na UFCG e ao CNPq por apoio à pesquisa do segundo e do terceiro autor.

\section{REFERÊNCIAS}

CEBALLOS JC. 1988. On two-flux approximations for shortwave radiative transfer in the atmosphere. Contributions to Atmospheric Physics, 61: 8-22.
CEBALLOS JC. 1989. Stochastic properties of two-flux shortwave radiative transfer in the atmosphere. Contributions to Atmospheric Physics, 62: 179-192.

CHANDRASEKHAR S. 1950. Radiative transfer. Oxford Press, Dover Publications, New York, 393 pp.

CHOU MD \& SUAREZ MJ. 1999. A Solar Radiation Parameterization for Atmospheric Studies. NASA/TM-1999-104606, Vol. 15.51 pp.

COAKLEY JA Jr \& CHYLEK PJ. 1975. The two stream Approximation in Radiative Transfer. Including the angle of the incident radiation. Journal of the Atmospheric Sciences, 32: 409-418.

COELHO PJ. 2005. Fundamentals of a new method for the solution of the radiative transfer equation. International Journal of Thermal Sciences 44: 809-821.

COX DR \& MILLER HD. 1967. The theory of stochastic processes. Chapman \& Hall, London, 398 pp.

DEIRMENDJIAN D \& SEKERA Z. 1954. Global radiation resulting from multiple scattering in a Rayleigh atmosphere. Tellus, VI: 382-398.

FILYUSHKIN VV, MADRONICHS, BRASEUR GP \& PETROPAVLOVSKIKH IV. 1994. Fast Two-Stream Method for Computing Diurnal-Mean Actinic Flux in Vertically Inhomogeneous Atmospheres. Journal of the Atmospheric Sciences, 51: 1077-1088.

FU Q, LIOU KN, CRIBB MC \& CHARLOCK TP. 1997. Multiple Scattering Parameterization in Thermal Infrared Radiative Transfer. American Meteorological Society, 54: 2799-2812.

GUEYMARD CA. 2008. REST2: High-performance solar radiation model for cloudless-sky irradiance, illuminance, and photosynthetically active radiation - Validation with a benchmark dataset. Solar Energy, 82: 272-285.

IQBAL M. 1983. An Introduction to solar radiation, Academic Press, Toronto, $391 \mathrm{pp}$.

JOSEPH JN, WISCOMBE WJ \& WEIMAN JA. 1976. The delta-Eddington approximation for radiative flux transfer. Journal of the Atmospheric Sciences, 33: 2452-2459.

KING MD \& HARSHVARDHAN. 1986. Comparative Accuracy of Selected Multiple Scattering Approximations. Journal of the Atmospheric Sciences, 43: 784-801.

LACIS AA \& HANSEN JE. 1974. A parameterization for the absorption of solar radiation in the Earth's atmosphere. Journal of the Atmospheric Sciences, 31: 118-133.

LECKNER B. 1978. The spectral distribution of solar radiation at the Earth's surface-elements of a model. Solar Energy, 20: 143-150.

LEIGHTON HG. 1980. Application of the delta-Eddington Method to the absorption of solar radiation in the atmosphere. Atmosphere-Ocean, 18: 43-52. 
LENOBLE J. 1993. Radiative transfer in scattering and absorbing atmospheres. A. Deepak Publishing, 532 pp.

LIOU KN. 1974. Analytic two-stream and four-stream solutions for radiative transfer. Journal of the Atmospheric Sciences, 31: 1473-1475.

LIOU KN. 1980. An introduction to atmospheric radiation. Academic Press California, $392 \mathrm{pp}$.

LIOU KN. 1992. Radiation and Cloud Processes in the Atmosphere, Oxford University Press. 487 pp.

MCCARTNEY EARL J. 1976. Optics of the atmosphere. Scattering by Molecules and Particles. John Wiley \& Sons, Inc. 408 pp.

McCLATCHEY RA, FENN RW, SELBY JEA, VOLZ EE \& GARING JS. 1971. Optical properties of the atmosphere. Environ. Res. Pap. 354. Air Force Cambridge Res. Lab. Bedford, Massachusetts. 85 pp.

MEADOR WE \& WEAVER WR. 1980. Two-stream approximations to radiative transfer in planetary atmospheres: A unified description of existing methods and a new improvement. Journal of the Atmospheric Sciences, 37: 630-643.

PALTRIDGE GW \& PLATT CMR. 1976. Radiative processes in meteorology and climatology. Elsevier, $318 \mathrm{pp}$.

RICCHIAZZI P, YANG S, GAUTIER C \& SOWLE D. 1998. SBDART: A Research and Teaching Software Tool for Plane-Parallel Radiative Transfer in the Earth's Atmosphere. Bulletin of the American Meteorological Society, 79: 2101-2113.

ROZANOV A, ROZANOV V, BUCHWITZ M, KOKHANOVSKY A \& BURROWS JP. 2005. SCIATRAN 2.0 - A new radiative transfer model for geophysical applications in the $175-2400 \mathrm{~nm}$ spectral region. Advances in Space Research, 36: 1015-1019.

SBDART. 2004. Input documentation for SBDART, version 2.4, august, 2004. Disponível em: < http://cygnus.atk.com/sbdart/html/sbdartdoc.txt> . Acesso em: 17 abr. 2007.

SCHMETZ J. 1984. On the parameterization of the radiative properties of broken clouds. Tellus, 36A: 417-432.

SCHALLER E. 1979. A delta two-stream approximation in radiative flux calculation. Contributions to Atmospheric Physics, 52: 17-26.

SHETTLE EP \& FENN RW. 1979. Models for the aerosol of the lower atmosphere and the effect of humidity variations on their optical properties. Environ. Res. Paper No. 676, Air for Force Geophysics Lab. Hanson AFB, Massachusetts, USA. 94 pp.

SHETTLE EP \& WEINMAN JA. 1970. The transfer of solar irradiance though inhomogeneous turbid. Atmospheric evaluated by Eddington's approximation. Journal of the Atmospheric Sciences, 27: 1048-1055.

STEPHENS GL. 1984. The parameterization of radiation for numerical weather prediction and climate models. Mon. Wea. Rev., 112: 826-867.
STEPHENS GL, GABRIEL PM \& PARTAIN PT. 2001. Parameterization of atmospheric radiative transfer. Part I: Validity of simple models. Journal of the Atmospheric Sciences, 58: 3391-3409.

SUBASILAR B. 2008. Analytical approaches to the delta-Eddington model of the radiative transfer through vertically inhomogeneous optical depths. Applied Mathematical Modelling, 32: 514-534.

ZDUNKOWSKI WG, WELCH R \& KORB G. 1980. An investigation of the structure of typical two-stream-methods for the calculation of solar fluxes and heating rates in clouds. Contributions to Atmospheric Physics, 53: 147-166.

ZHANG F, ZENG Q, GU Y \& LIOU KN. 2005. Parameterization of the absorption of $\mathrm{H}_{2} \mathrm{O}$ continuum, $\mathrm{CO}_{2}, \mathrm{O}_{2}$, and other trace gases in the Fu-Liou solar radiation program. Advances in Atmospheric Sciences, 22: $545-558$

ZORZANO MP, MANCHO AMB \& VAZQUEZ LV. 2005. Numerical integration of the discrete-ordinate radiative transfer equation in strongly nonhomogeneous media discrete-ordinate. Applied Mathematics and Computation, 164: 263-274.

\section{APÊNDICE A}

\section{Soluções do modelo de dois fluxos para uma camada}

No sistema de Eqs. (1) define-se a irradiância relativa a incidente no topo, $M^{\downarrow \uparrow}(\tau)$, como:

$$
\begin{gathered}
M^{\downarrow \uparrow}(\tau)=\frac{E^{\downarrow \uparrow}(\tau)}{\mu_{0} S_{0, \lambda}}, \\
M_{0, \lambda}(\tau)=\omega \frac{S_{\lambda}(\tau)}{\mu_{0} S_{0, \lambda}}=\omega m_{0} e^{-\tau m_{0}} ; \quad m_{0}=\frac{1}{\mu_{0}}, \\
A(\alpha)=\left[\begin{array}{cc}
-\alpha_{1} & \alpha_{2} \\
-\alpha_{2} & \alpha_{1}
\end{array}\right], \\
A\left(b_{0}\right)=\left[\begin{array}{c}
1-b\left(\mu_{0}\right) \\
b\left(\mu_{0}\right)
\end{array}\right],
\end{gathered}
$$

tem-se:

$$
\begin{array}{r}
\frac{d}{d \tau}\left[\begin{array}{c}
M^{\downarrow} \\
M^{\uparrow}
\end{array}\right](\tau)= \\
+M_{0, \lambda}(\tau) A\left(b_{0}\right) .
\end{array}
$$

Na solução do sistema de Eqs. (A5), observa-se:

1) Condições de contorno:

a) No topo da camada:

$$
M^{\downarrow \uparrow}(0)=\left(\begin{array}{c}
0 \\
R p
\end{array}\right) .
$$


b) Na base da camada:

$$
M^{\uparrow}(\tau)=R s\left[M^{\downarrow}(\tau)+e^{-\tau m_{0}}\right] .
$$

2) 0 fluxo que incide na camada atmosférica pode ser direto ou difuso e tem a possibilidade de ser absorvido, transmitido ou refletido. 0 fluxo direto pode interagir com os componentes atmosféricos no interior da camada e tornarse difuso. Considera-se que a refletância do solo $R s=0$.

3) A solução depende do tipo de atmosfera: a) conservativa (atmosfera Rayleigh, $\omega=1$ ), onde 0 autovalor do sistema (Eqs. A5) torna-se nulo e b) não conservativa $(\omega<1)$, em que 0 autovalor é diferente de zero. Por conseguinte, essas condições conduzem a duas soluções distintas. Neste trabalho, no caso de uma atmosfera conservativa, assume-se que $\omega=0,999999$, o que permite usar uma única solução para camadas atmostéricas homogêneas.

Se na camada incide apenas fluxo difuso, então a solução do sistema de Eqs. (A5) tem a forma:

$$
\begin{aligned}
& M^{\downarrow}(\tau)=a_{1}(\tau) \operatorname{Rp}(\tau), \\
& M^{\uparrow}(\tau)=b_{1}(\tau) \operatorname{Rp}(\tau),
\end{aligned}
$$

onde:

$$
\begin{gathered}
a_{1}(\tau)=\frac{e^{\lambda \tau}-e^{-\lambda \tau}}{x-y}, \\
b_{1}(\tau)=\frac{x e^{\lambda \tau}-y e^{-\lambda \tau}}{x-y}, \\
c_{1}(\tau)=\frac{y e^{\lambda \tau}-x e^{-\lambda \tau}}{x-y}, \\
x=\frac{\alpha_{1}+\lambda}{\alpha}, \quad y=\frac{\alpha_{1}-\lambda}{\alpha_{2}}, \\
\lambda= \pm \sqrt{\alpha_{1}^{2}-\alpha_{2}^{2}} .
\end{gathered}
$$

As transições intrínsecas da camada para fluxo ascendente e descendente obedecem às relações: $T^{\downarrow}(\tau)=T^{\uparrow}(\tau) \mathrm{e}$ $R^{\downarrow}(\tau)=R^{\uparrow}(\tau)$, de forma que:

$$
\begin{gathered}
T(\tau)=\frac{M^{\uparrow}(0)}{M^{\uparrow}(\tau)}=\frac{1}{b_{1}(\tau)}, \\
R(\tau)=\frac{M^{\downarrow}(\tau)}{M^{\uparrow}(\tau)}=\frac{a_{1}(\tau)}{b_{1}(\tau)}, \\
A(\tau)=1-R(\tau)-T(\tau)=1-\frac{1+a_{1}(\tau)}{b_{1}(\tau)} .
\end{gathered}
$$

Se no topo da camada incide fluxo direto, então a solução do sistema de Eqs. (A5) corresponde a solução do sistema para fluxos difusos, acrescido de uma solução particular $M p$, correspondente a fluxo direto. Fazendo:

$$
M p^{\downarrow}(\tau)=-\gamma_{1} e^{-\tau m_{0}} e M p^{\uparrow}(\tau)=-\gamma_{2} e^{-\tau m_{0}}
$$

e combinando as condições de contorno e a solução particular no sistema de Eqs. (A5), tem-se:

$$
\begin{aligned}
& M^{\downarrow}(\tau)=a_{1}(\tau) \operatorname{Rp}(\tau)+a_{2}(\tau)-a_{3}(\tau), \\
& M^{\uparrow}(\tau)=b_{1}(\tau) \operatorname{Rp}(\tau)+b_{2}(\tau)-b_{3}(\tau),
\end{aligned}
$$

onde:

$$
\begin{gathered}
R p(\tau)= \\
=\frac{R s\left[a_{2}(\tau)-a_{3}(\tau)+e^{-\tau m_{0}}\right]-b_{2}(\tau)+b_{3}(\tau)}{b_{1}(\tau)-a_{1}(\tau) R s}, \\
a_{2}(\tau)=-\gamma_{1} c_{1}(\tau)+\gamma_{2} a_{1}(\tau) ; \\
a_{3}(\tau)=\gamma_{1} e^{-\tau m_{0}}, \\
b_{2}(\tau)=-\gamma_{1} a_{1}(\tau)+\gamma_{2} b_{1}(\tau) ; \\
b_{3}(\tau)=\gamma_{2} e^{-\tau m_{0}}, \\
\gamma_{1}=\frac{\omega m_{0}\left[\left(1-b_{0}\right)\left(m_{0}+\alpha_{1}\right)+\alpha_{2} b_{0}\right]}{m_{0}^{2}-\lambda^{2}}, \\
\gamma_{2}=\frac{\omega m_{0}\left[-b_{0}\left(m_{0}-\alpha_{1}\right)+\alpha_{2}\left(1-b_{0}\right)\right]}{m_{0}^{2}-\lambda^{2}} .
\end{gathered}
$$

Considerando uma atmosfera de uma única camada com espessura óptica $\tau$ entre 0 topo da atmosfera e a superfície, a refletância planetária $(R p)$ é dada pela Eq. (A21) e corresponde a $M^{\uparrow}(0)$ na Eq. (A20). As frações intrínsecas de fluxos da camada, referentes à refletância $R(\tau)$, transmitância $T(\tau)$ e absortância $A(\tau)$, são estimadas conforme as Eqs. (A15)-(A17).

Na superfície as frações referentes à transmitância difusa $M_{D}(\tau)$, direta $M_{D R}(\tau)$, global $M_{g}(\tau)$ e absortância $A(\tau)$, são estimadas conforme as Eqs. (A19)-(A21), onde:

$$
\begin{gathered}
M_{D}(\tau)=M^{\downarrow}(\tau)-M^{\uparrow}(\tau), \\
M_{D R}(\tau)=\exp \left(-m_{0} \tau\right), \\
M_{g}(\tau)=M_{D}(\tau)+M_{D R}(\tau), \\
A(\tau)=1-M_{g}(\tau)-R p(\tau) .
\end{gathered}
$$




\section{APÊNDICE B}

$\mathrm{Na}$ Eq. (19) os elementos das matrizes $U, V$ e $Q$ são deduzidos a partir da Figura 1. Os elementos dessas matrizes são constituídos por frações de fluxos estritamente difusos. Para um número $N$ de camadas e um número $N A=N+2$ de estados absorventes, os elemento não nulos $u(i, j)$ e $v(i, j)$ das respectivas matrizes $U$ e $V$, são determinados observando as transições de fluxos difusos na Figura 1.

Os elementos $u(i, j)$ não nulos da matriz $U$ são:

Para $1 \leq i \leq 2 N$ e $1 \leq j \leq N A$ :

$$
\begin{gathered}
u(2,1)=T_{D F}\left(\tau_{1}\right), \\
u(2 N, N A)=A\left(\tau_{N}\right), \\
u(2 N-1,2)=1-R s .
\end{gathered}
$$

Se $j \leq N-1$ :

$$
\begin{gathered}
u(2 j, j+2)=A(j), \\
u(2 j-1, j+3)=A(j+1) .
\end{gathered}
$$

Como exemplo, conforme a Figura 1, $u(2,1)$ corresponde a transição do estado 20 ao estado $1 ; u_{(2 N, N A)}$ corresponde a transição do estado 49 ao estado 2; $u_{3(2 N-1,2)}$ corresponde a transição do estado 49 ao estado 2.

Os elementos não nulos da matriz $V$ são:

Para $1 \leq i \leq 2 N$ e $1 \leq j \leq 2 N$ :

$$
\begin{gathered}
v(2 N, 2 N-2)=T_{D}\left(\tau_{N}\right) . \\
v(2 N-1,2 N)=R s . \\
v(2 N, 2 N-1)=R_{D}\left(\tau_{N}\right) .
\end{gathered}
$$

Se $j \leq N-1$ então:

$$
\begin{gathered}
v(2 j, 2 j-1)=R_{D}\left(\tau_{j}\right) . \\
v(2 j+2,2 j)=T_{D}\left(\tau_{j+1}\right) . \\
v(2 j-1,2 j)=R_{D}\left(\tau_{j+1}\right) . \\
v(2 j-1,2 j+1)=T_{D}\left(\tau_{j+1}\right) .
\end{gathered}
$$

Como exemplo, conforme a Figura 1, $v(2 N, 2 N-2)$ corresponde a transição do estado 50 ao estado 48.

\section{NOTAS SOBRE OS AUTORES}

Juarez Dantas de Souza concluiu o curso de Licenciatura Plena em Matemática no ano de 1978 na Universidade Regional do Nordeste, hoje Universidade Estadual da Paraíba - UEPB. Concluiu o curso de Mestrado em Meteorologia na Universidade Federal da Paraíba - UFPB no ano de 1995. É professor efetivo de matemática da Universidade Estadual da Paraíba - UEPB e doutorando do Programa de Pós-graduação em Meteorologia da Universidade Federal de Campina Grande.

Bernardo Barbosa da Silva possui Mestrado em Meteorologia e Doutorado em Recursos Hídricos, ambos na Universidade Federal da Paraíba - UFPB, e Pósdoutorado na Universidade do Arizona. Atua nos cursos de graduação e pós-graduação em Meteorologia (níveis mestrado e doutorado), é pesquisador do CNPq e tem orientado estudantes de graduação e pós-graduação. Já publicou cerca de 40 artigos em revistas científicas nacionais e internacionais, e concluiu a orientação de 24 mestres e 10 doutores.

Juan Carlos Ceballos é graduado em Física pela Universidad Nacional de Tucumán, Argentina (1966). Doutor em Meteorologia pelo Instituto Astronômico e Geofísico da Universidade de São Paulo, Brasil (1986). Estágio de pesquisa em propagação da radiação solar em nuvens finitas no Laboratoire d’Optique Atmosphérique, Université de Lille, Franç̧a. Professor aposentado do Departamento de Ciências Atmosféricas, Centro de Ciências e Tecnologia, Universidade Federal da Paraíba. Pesquisador na Divisão de Satélites e Sistemas Ambientais, Centro de Previsão de Tempo e Estudos Climáticos, Instituto Nacional de Pesquisas Espaciais. 